\title{
Assessing the risk of observing multiple generations of Middle East respiratory syndrome (MERS) cases given \\ an imported case
}

H Nishiura (nishiurah@gmail.com) ${ }^{1,2}$, Y Miyamatsu ${ }^{1,2}$, G Chowell 3,4, M Saitoh ${ }^{1,2,5}$

1. Graduate School of Medicine, The University of Tokyo, Tokyo, Japan

2. CREST, Japan Science and Technology Agency, Saitama, Japan

3. School of Public Health, Georgia State University, Atlanta, Georgia, United States

4. Division of International Epidemiology and Population Studies, Fogarty International Center, National Institutes of Health,

5. Bethesda, Maryland, United States

6. The Institute of Statistical Mathematics, Tachikawa, Japan

Nishiura H, Miyamatsu Y, Chowell G, Saitoh M. Assessing the risk of observing multiple generations of Middle East respiratory syndrome (MERS) cases given an imported case. Euro Surveill. 2015;20(27):pii=21181. Available online: http://www.eurosurveillance.org/ViewArticle.aspx?Articleld=21181

To guide risk assessment, expected numbers of cases and generations were estimated, assuming a case importation of Middle East respiratory syndrome (MERS). Our analysis of 36 importation events yielded the risk of observing secondary transmission events at $\mathbf{2 2 . 7} \%$ (95\% confidence interval: $19.3-25.1)$. The risks of observing generations 2, 3 and 4 were estimated at $10.5 \%, 6.1 \%$ and $3.9 \%$, respectively. Countries at risk should be ready for highly variable outcomes following an importation of MERS.

Middle East respiratory syndrome (MERS) has continued to spread globally [1]. A large cluster of MERS cases has been observed in the Republic of Korea since May 2015 [2]. Until 1 July 2015, so-called quaternary cases (i.e. generation 3 counting from the index case as generation o) have been reported in South Korea [2]. Although the inter-human transmission potential of MERS is considered to be too low to cause large-scale epidemics [3-5], high variability and heterogeneity in the transmission potential have been underscored [6]. As MERS continues to spread globally, it is vital that risk assessment involves an evaluation of the potential outcomes following MERS importation events [7]. Among a total of 23 importation events in countries outside the Middle East region, there have been four MERS case importations that have given rise to at least one secondary transmission event [2].

While the basic reproduction number $R_{0}$, i.e. the average number of secondary cases produced by a primary case in a fully susceptible population, is less than 1 for MERS and a major epidemic may therefore not occur immediately, it is critical to quantitatively assess several risks of MERS transmission following an importation event, e.g. the expected numbers of cases and generations. The present study aims to analyse the observed importation events of MERS and estimate the expected size of MERS clusters and the number of generations using a stochastic epidemic model.

\section{Importation data}

Using secondary data sources [2,7-11], we extracted the numbers of secondary cases and generations for each reported importation event of MERS. We excluded data from the Kingdom of Saudi Arabia, Qatar and the United Arab Emirates from the analysis because cases in these endemic countries have frequently experienced exposures to a domestic animal reservoir (e.g. dromedary camels). Moreover, it has not been possible to fully track the transmission trees (i.e. via contact tracing data) in these countries. Including Middle East countries other than those, a total of 36 importation events were analysed. Of these, 13 events occurred in the Middle East, reported from Egypt, Iran, Jordan, Kuwait, Lebanon, Oman, Turkey and Yemen. In the present study, statistical estimation of parameters was done using two different sets of data, i.e. using all 36 importation events and restricting the analysis to the 23 importation events observed in areas outside of the Middle East. The latter data were analysed separately because the dynamics of case importation including the frequency of exposures and local contact patterns for those 23 events may not be fully comparable with the remaining 13 events.

\section{Mathematical model}

The importation event data were analysed using a branching process model which allowed us to jointly estimate the transmissibility, $R_{0}$, and the dispersion parameter, $k$, by assuming that the distribution of secondary cases per single primary case followed a 
negative binomial distribution. Using the branching process model, the risk of observing at least one secondary transmission event, the risk of observing each generation of cases and the total number of cases were estimated. Hence, an important assumption to calculate these risks based on observed importation event data is that each importation event was a random sample drawn from the assumed model. For the estimation of the two model parameters, we used two different pieces of likelihood. The first one analyses the distribution of the total number of cases as described by Breban et al. for MERS [4], but the present study specifically focused on importation events [12]. Given the total number of cases $z$ for each importation event, the likelihood to estimate $R_{\mathrm{o}}$ and $k$ was calculated as

Equation 1:

$$
L_{1}\left(R_{0}, k ; z\right)=\left\{\begin{array}{c}
\frac{1}{\left(1+\frac{R_{0}}{k}\right)^{k}, \text { for } z=1} \\
\frac{\prod_{j=0}^{z-2}\left(\frac{j}{k}+z\right)}{z !}\left(\frac{1}{1+\frac{R_{0}}{k}}\right)^{z k}\left(\frac{R_{0}}{1+\frac{R_{0}}{k}}\right)^{z-1} \quad \text { for } z \geq 2
\end{array}\right.
$$

The number of observed importation events was limited to 36 and the sample size was small. To reduce uncertainty, we analysed in addition the number of generations per importation. Considering the concept of generation to extinction, we derived the probability distribution of the number of generations, i.e.

Equation 2:

$$
\operatorname{Pr}(g \leq h)=\left\{\begin{array}{c}
\frac{1}{\left(1+\frac{R_{0}}{k}\right)^{k}}, \text { for } h=1 \\
\frac{1}{\left(1+\frac{R_{0}}{k}-\frac{R_{0}}{k\left(1+\frac{R_{0}}{k}\right)^{k}}\right)^{k}}, \text { for } h=2 \\
\frac{1}{\left(1+\frac{R_{0}}{k}(1-\operatorname{Pr}(g \leq h-1))\right)^{k}}-\text { for } h \geq 3
\end{array}\right.
$$

from which we obtained the likelihood function $L_{2}$ for the observed number of generations, derived from $\operatorname{Pr}(g \leq h)-\operatorname{Pr}(g \leq h-1)$ for $h>1$, i.e.

and so forth. The total likelihood was given by the product of $L_{1}$ and $L_{2}$. The maximum likelihood method was employed to statistically infer the parameters, and the profile likelihood-based confidence intervals (Cl) were computed. To account for both stochasticity and parameter uncertainty in calculating the outbreak size

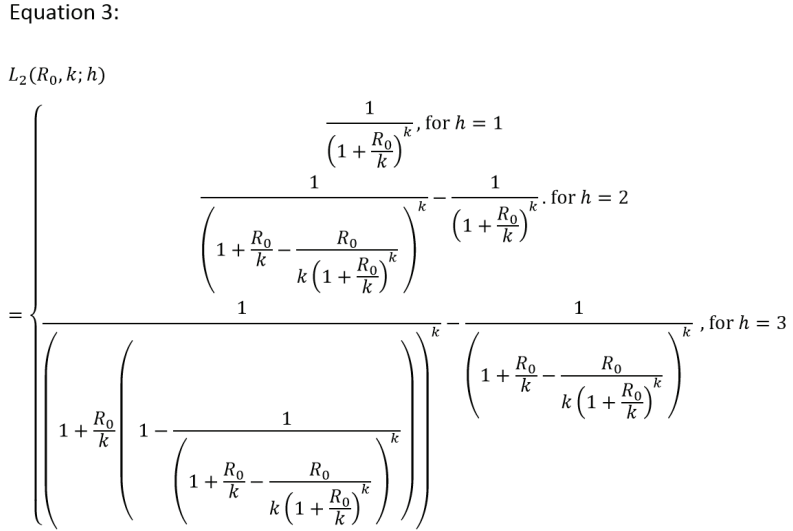

distribution and the risk of observing each generation of cases, the covariance matrix was used to draw random samples of $R_{0}$ and $k$ from a bivariate normal distribution with the correlation parameter informed by the matrix. The simulations were run 10,000 times, allowing us to take percentile points for the calculation of uncertainty bounds.

\section{Results}

Figure 1 illustrates the original data employed to quantify our model.

The distribution of the total number of cases was right skewed, including two outbreaks with a total of at least eight cases. Excluding the Middle East countries, only the ongoing South Korean outbreak was the one that involved eight or more cases. With regard to the number of generations, three quarters of the importations (27/36) did not result in any secondary transmission. Secondary transmissions were observed in Iran, Jordan, Kuwait, France, Republic of Korea, Oman (twice), Tunisia and the United Kingdom. Excluding Middle East countries, 19 of 23 importations did not generate any secondary cases (Table 1). Among the total of 36 importations, generation 3 (or the so-called quaternary cases) was observed only for the ongoing South Korean outbreak.

Table 2 shows the estimates of $R_{0}$ and $k$. Based on a total of 36 events, $R_{0}$ and $k$ were estimated at 0.75 (95\% Cl: $0.54-1.09)$ and $0.14(95 \% \mathrm{Cl}: 0.06-0.32)$, respectively.

The value of $k$ was substantially smaller than 1 , indicating that the distribution of secondary cases per single primary case was highly over-dispersed. Analysing the 23 importation events out of the Middle East countries, the estimates of $R_{\circ}$ and $k$ were $0.81(95 \% \mathrm{Cl}$ : $0.49-1.46)$ and 0.07 (95\% Cl: $0.02-0.21)$, respectively. These estimates were not significantly different from those obtained using the total set of 36 importation events. For this reason, simulations were conducted using estimates derived from the full set of 36 importation events. 


\section{FIGURE 1}

Importation events of Middle East respiratory syndrome $(\mathrm{n}=36)$

A.

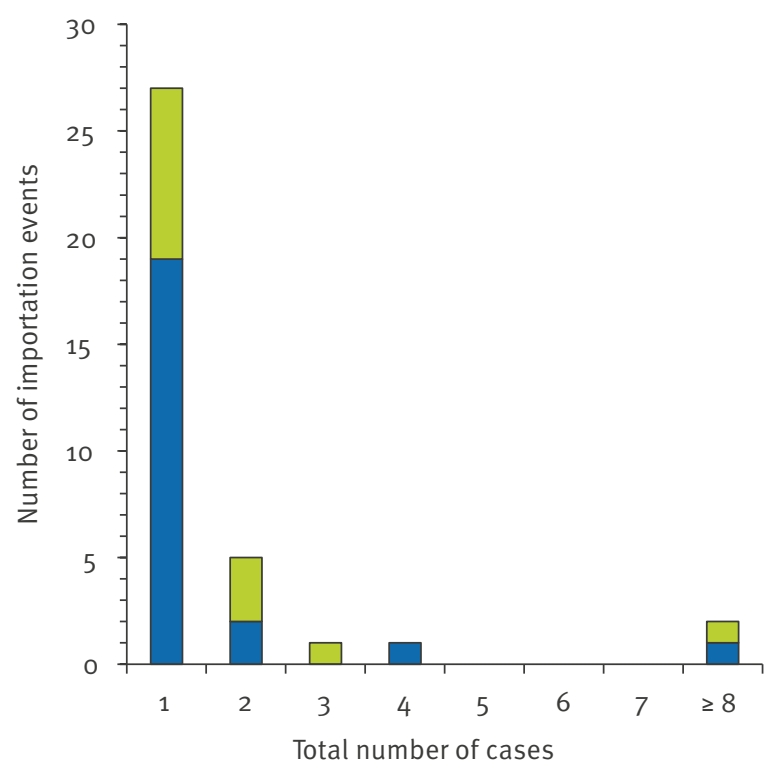

B.

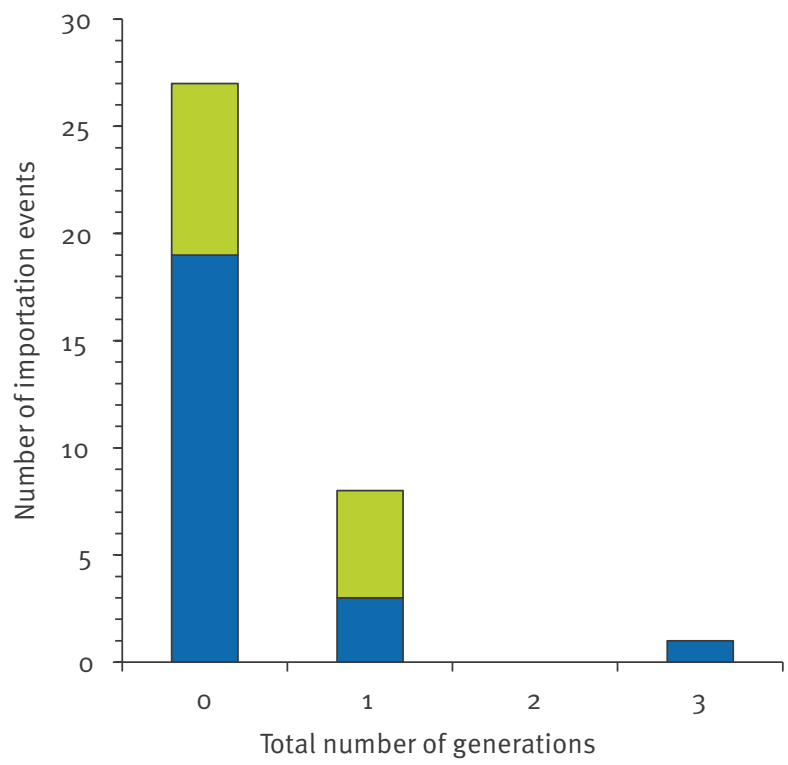

Middle East countries

Other than Middle East countries

A. The observed number of importation events as a function of the total number of cases. An importation event frequently ends up with only the imported (index) case, i.e. without generating any secondary cases.

B. Observed number of importation events by total number of generations observed for each importation. Generation o represents the imported (index) case, generation 1 represents secondary cases produced by the imported case, and so forth.

\section{TABLE 1}

Importation of Middle East respiratory syndrome to the 23 countries outside the Middle East that have experienced case until 1 July 2015 ( $\mathrm{n}=208$ cases)

\begin{tabular}{|l|c|c|}
\hline Country & Generation & Total number of cases \\
\hline Algeria & 0 & 1 \\
\hline Algeria & 0 & 1 \\
\hline Austria & 0 & 1 \\
\hline China & 0 & 1 \\
\hline France & 1 & 2 \\
\hline Germany & 0 & 1 \\
\hline Germany & 0 & 1 \\
\hline Germany & 0 & 1 \\
\hline Germany & 0 & 1 \\
\hline Greece & 0 & 1 \\
\hline Italy & 0 & 1 \\
\hline Malaysia & 0 & 1 \\
\hline The Netherlands & 0 & 1 \\
\hline The Netherlands & 0 & 1 \\
\hline Philippines & 0 & 1 \\
\hline Philippines & 0 & 181 \\
\hline South Korea & 3 & 1 \\
\hline Thailand & 0 & 1 \\
\hline Tunisia & 0 & 1 \\
\hline Tunisia & 0 & 1 \\
\hline United Kingdom & 0 & 1 \\
\hline United States & 0 & 1 \\
\hline United States & 0 & 1 \\
\hline
\end{tabular}

The generation column represents the total number of generations (e.g. o indicates that the imported index case did not generate any secondary cases), while the total number of cases represents the cumulative number of confirmed cases including the index case

(e.g. 1 indicates that the imported index case did not generate any secondary cases). For instance, in France, there was a secondary transmission, causing generation 1 , but there was only 1 secondary case without tertiary case, and thus, the total number of cases was 2.

According to our classification, Turkey was included in the Middle East.

Figure 2 shows the expected total number of cases and the risk of observing each generation conditional on an importation event.

The risk of observing at least one secondary transmission was $22.7 \%(95 \% \mathrm{Cl}$ : 19.3-25.1) (Figure 2A). The risks of observing generations 2, 3 and 4 were, respectively, $10.5 \%, 6.1 \%$ and $3.9 \%$ (Figure 2B). When generation 2 (tertiary cases) was observed, the conditional risk of observing the next generation (quaternary cases) was as large as $63.6 \%(95 \% \mathrm{Cl}: 46.7-74.4)$ (Figure $2 \mathrm{C}$ ). The outbreak size distribution appeared to be highly skewed (Figure 2D). Assuming an importation occurred, the risk of observing eight or more cases in total would be $10.9 \%$ ( $95 \% \mathrm{Cl}: 7.6-13.6)$.

\section{Discussion}

The present study analysed importation events of MERS with a particular focus on the associated outbreak size 


\section{FIGURE 2}

Probabilities of observing multiple generations of Middle East respiratory syndrome cases given one case importation

A.

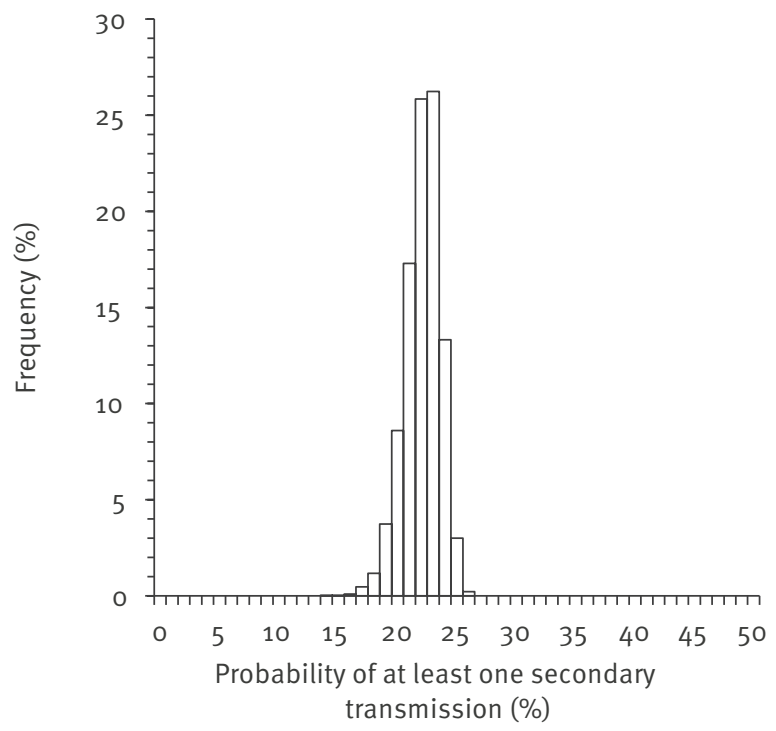

C.

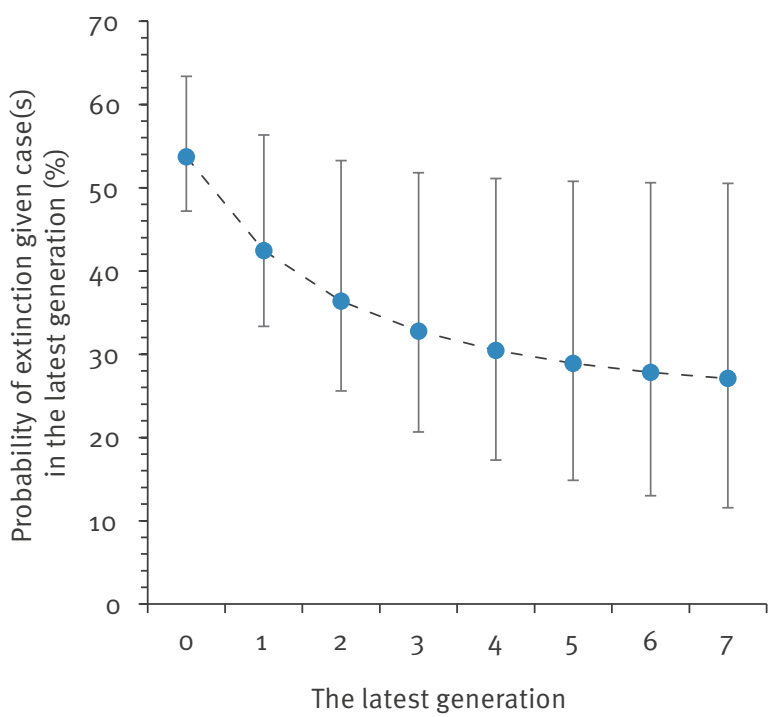

B.

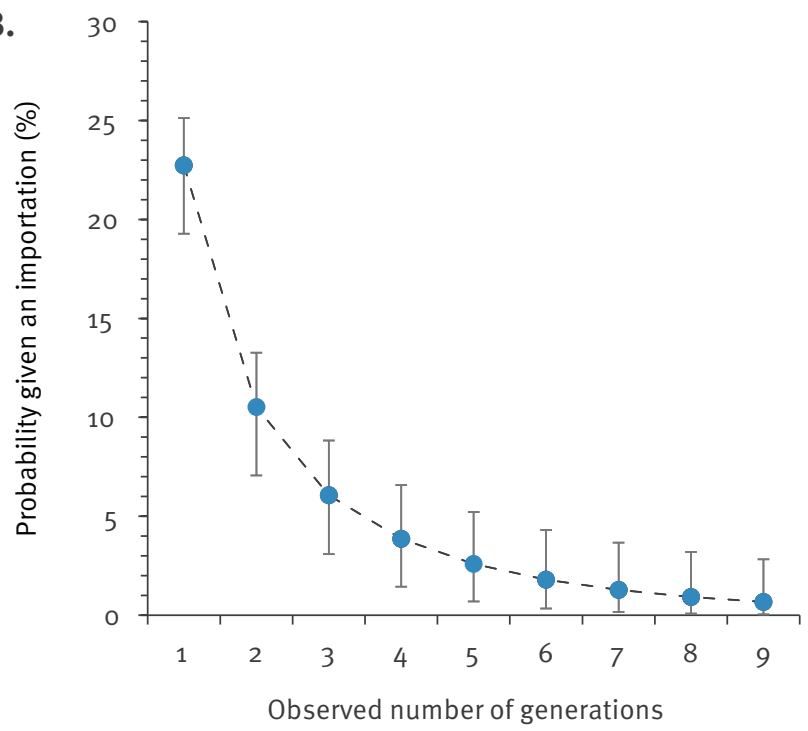

D.

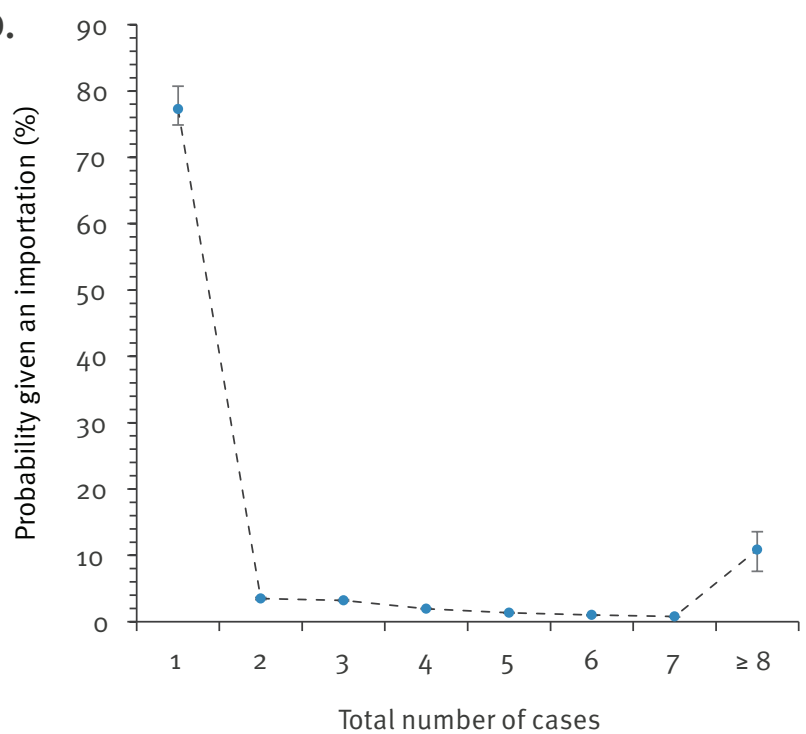

A. Frequency distribution of the probability of observing at least one secondary case caused by an imported (index) case, based on a total of 10,000 simulation runs.

B. Probability that the transmission survives to a specific generation given an imported (index) case.

C. Conditional probability that the transmission goes extinct at the particular generation given case(s) in the corresponding generation. Until 20 June 2015 , cases of MERS up to generation 3 (i.e. or the so-called quaternary cases) have been diagnosed in South Korea.

D. Frequency distribution of the total number of cases given an imported case.

In panels B, C and D, filled circles represent the posterior median of simulations, while whiskers extend to upper and lower $95 \%$ credible intervals, based on 10,000 posterior resampling simulation runs.

and number of disease generations arising from each importation event. Our findings indicate that if a case of MERS was imported, at least one secondary transmission event would be observed with a probability of $22.7 \%$. The risk of involving tertiary, quaternary and quinary cases was also calculated (Figure 2B). Although our study only relied upon importation events consisting of a small number of clusters, the estimated $R_{\text {o }}$ was broadly consistent with published estimates that analysed larger cluster datasets mainly observed in Middle East countries [3-5] and was smaller than estimates derived from the early phase of hospitalassociated outbreaks [13]. Considering that countries at risk of importation (i.e. countries without infected animal reservoirs) have had to confront the uncertainty associated with the risk of experiencing a case importation $[14,15,16]$, our modelling analyses could facilitate quantitative risk assessment.

An important outcome of the present study is that the risk of observing multiple generations of MERS cases was estimated at $22.7 \%$ and that of a tertiary case at 
Estimated transmission potential and dispersion parameter of Middle East respiratory syndrome based on imported case data

\begin{tabular}{|l|c|c|} 
& $\begin{array}{c}\text { Basic reproduction number } \\
(95 \% \mathrm{Cl})^{\mathrm{a}}\end{array}$ & $\begin{array}{c}\text { Dispersion parameter } \\
(95 \% \mathrm{Cl})\end{array}$ \\
\hline All importation events $(\mathrm{n}=36)$ & $0.75(0.54-1.09)$ & $0.14(0.06-0.32)$ \\
\hline Importation events in countries other than Middle East $(\mathrm{n}=23)^{\mathrm{b}}$ & $0.81(0.49-1.46)$ & $0.07(0.02-0.21)$ \\
\hline
\end{tabular}

$\mathrm{Cl}$ : confidence interval.

a $95 \%$ confidence intervals were derived from the profile likelihood.

${ }^{\mathrm{b}}$ Excluded Middle East countries are: Egypt, Iran, Jordan, Kuwait, Lebanon, Oman, Turkey and Yemen.

$10.5 \%$ in our model. Since the distribution of secondary cases per single primary case is highly over-dispersed, superspreading events can occur, and thus, the expected total number of cases is highly variable. The finding echoes a recent re-analysis of clusters of MERS cases reported up to August 2013 [17]. Of course, we can expect that secondary transmission events could be prevented by a combination of contact tracing, monitoring suspected cases, early diagnosis and isolation of infectious individuals. Besides, the present study suggests that countries at risk of importation should keep in mind that a large cluster of cases with multiple generations may well occur, even though $R_{0}$ is clearly below the epidemic threshold at 1.0 [18].

In addition to the risk of observing a certain number of generations following an imported case, we also calculated the conditional risk of observing the next generation of case(s). This is in line with a risk assessment in real time: because the exact number of cases in a single generation cannot be manually counted during the course of an outbreak, it is reasonable to calculate the conditional risk given a generation where the conditional probability of observing the next generation is usually greater than $50 \%$.

Two important limitations should be noted. Firstly, our exercise regarded each observed importation event as a random draw governed by the proposed probability model. Indeed, this assumption is unavoidable for fitting a branching process model to the data $[3,4]$. While the assumption may be common among modelling studies, it did not allow us to account for the variable type and effectiveness of interventions, especially at later generations of cases in each cluster. Secondly, MERS outbreaks have frequently been amplified in healthcare settings $[6,19,20]$, but we limited ourselves to accounting for individual heterogeneity in a general sense. An improvement on this point was difficult, because MERS outbreaks have been seen mostly in healthcare settings without large-scale community transmission. The transmission dynamics in and out of healthcare settings have not been consistently characterised across individual outbreaks of MERS.
Despite these limitations, the present study successfully characterised the risk of observing a certain number of cases and generations given a case importation of MERS. The risk of at least one secondary case in our model was $22.7 \%$, and that of a tertiary case was $10.5 \%$. Once an untraced case is imported, countries at risk should assume that the secondary transmission risk as well as the risk of observing multiple generations of cases exists and should be ready to respond effectively by following their preparedness plans to manage emerging infectious diseases.

\section{Acknowledgements}

$\mathrm{HN}$ received funding support from the Japan Society for the Promotion of Science (JSPS) KAKENHI Grant Numbers 26670308 and 26700028 , Japan Agency for Medical Research and Development, the Japan Science and Technology Agency (JST) CREST program and RISTEX program for Science of Science, Technology and Innovation Policy. GC acknowledge financial support from the Division of International Epidemiology and Population Studies, The Fogarty International Center, United States National Institutes of Health, funded in part by the Office of Pandemics and Emerging Threats at the United States Department of Health and Human Services, as well as support from grants NSF grant 1414374 as part of the joint NSF-NIH-USDA Ecology and Evolution of Infectious Diseases programme, United Kingdom Biotechnology and Biological Sciences Research Council grant BB/Moo8894/1 and NSF-IIS Grant\#1518939. The funders had no role in study design, data collection and analysis, decision to publish, or preparation of the manuscript.

\section{Conflict of interest}

None declared.

\section{Authors' contributions}

HN conceived mathematical modelling method and analysed the data. HN, YM, GC and MS drafted and revised the manuscript.

\section{References}

1. Zumla A, Hui DS, Perlman S. Middle East respiratory syndrome. Lancet. 2015. Corrected proofs in press. http://dx.doi. org/10.1016/S0140-6736(15)60454-8 
2. World Health Organization (WHO). Global Alert and Response (GAR). Coronavirus infections. Geneva: WHO. [Accessed: 22 Jun 2015]. Available from: http://www.who.int/csr/don/archive/ disease/coronavirus_infections/en/

3. Cauchemez S, Fraser C, Van Kerkhove MD, Donnelly CA Riley S, Rambaut A, et al. Middle East respiratory syndrome coronavirus: quantification of the extent of the epidemic, surveillance biases, and transmissibility. Lancet Infect Dis. 2014;14(1):50-6. http://dx.doi.org/10.1016/S14733099(13)70304-9 PMID:24239323

4. Breban R, Riou J, Fontanet A. Interhuman transmissibility of Middle East respiratory syndrome coronavirus: estimation of pandemic risk. Lancet. 2013;382(9893):694-9. http://dx.doi. org/10.1016/S0140-6736(13)61492-o PMID:23831141

5. Chowell G, Blumberg S, Simonsen L, Miller MA, Viboud C. Synthesizing data and models for the spread of MERS-CoV, 2013: key role of index cases and hospital transmission. Epidemics. 2014;9:40-51. http://dx.doi.org/10.1016/j. epidem.2014.09.011 PMID:25480133

6. Fisman DN, Leung GM, Lipsitch M. Nuanced risk assessment for emerging infectious diseases. Lancet. 2014;383(9913):18990. http://dx.doi.org/10.1016/S0140-6736(13)62123-6 PMID:24439726

7. European Centre for Disease Prevention and Control (ECDC). Severe respiratory diseases associated with Middle East respiratory syndrome coronavirus (MERS-CoV). Fifteenth update -8 March 2015. Stockholm: ECDC, 2015. Available from: http://ecdc.europa.eu/en/publications/Publications/ MERS_update_08-Mar2014.pdf

8. Thomas HL, Zhao H, Green HK, Boddington NL, Carvalho CF, Osman HK, et al. Enhanced MERS coronavirus surveillance of travelers from the Middle East to England. Emerg Infect Dis. 2014;20(9):1562-4. http://dx.doi.org/10.3201/eid2009.140817 PMID:25148267

9. Yavarian J, Rezaei F, Shadab A, Soroush M, Gooya MM, Azad TM. Cluster of Middle East respiratory syndrome coronavirus infections in Iran, 2014. Emerg Infect Dis. 2015;21(2):362-4. http://dx.doi.org/10.3201/eid2102.141405 PMID:25626079

10. Al-Abaidani IS, Al-Maani AS, Al-Kindi HS, Al-Jardani AK, AbdelHady DM, Zayed BE, et al. Overview of preparedness and response for Middle East respiratory syndrome coronavirus (MERS-CoV) in Oman. Int J Infect Dis. 2014;29:309-10. http:// dx.doi.org/10.1016/j.ijid.2014.10.003 PMID:25447719

11. Hijawi B, Abdallat M, Sayaydeh A, Alqasrawi S, Haddadin $A$, Jaarour $N$, et al. Novel coronavirus infections in Jordan, April 2012: epidemiological findings from a retrospective investigation. East Mediterr Health J. 2013;19(Suppl 1):S12-8. PMID:23888790

12. 12. Nishiura H, Yan P, Sleeman CK, Mode CJ. Estimating the transmission potential of supercritical processes based on the final size distribution of minor outbreaks. J Theor Biol. 2012;294:48-55. http://dx.doi.org/10.1016/j.jtbi.2011.10.039 PMID:22079419

13. Majumder MS, Rivers C, Lofgren E, Fisman D. Estimation of MERS-Coronavirus Reproductive Number and Case Fatality Rate for the Spring 2014 Saudi Arabia Outbreak: Insights from Publicly Available Data. PLoS Curr. 2014;6: pii:ecurrents.out breaks.98d2f8f3382d84f390736cd5f5fe133c. http://dx.doi. org/10.1371/currents.outbreaks.98d2f8f3382d84f390736cd5f5 fe133C PMID: 25685622

14. Poletto C, Gomes MF, Pastore y Piontti A, Rossi L, Bioglio L, Chao DL, et al. Assessing the impact of travel restrictions on international spread of the 2014 West African Ebola epidemic. Euro Surveill. 2014;19(42):20936. http://dx.doi. org/10.2807/1560-7917.ES2014.19.42.20936 PMID:25358040</

15. Nishiura H, Mizumoto K, Ejima K, Zhong Y, Cowling B, Omori $R$. Incubation period as part of the case definition of severe respiratory illness caused by a novel coronavirus. Euro Surveill. 2012;17(42):20296. PMID:23098822

16. Ejima K, Aihara K, Nishiura H. Probabilistic differential diagnosis of Middle East respiratory syndrome (MERS) using the time from immigration to illness onset among imported cases. J Theor Biol. 2014;346:47-53. http://dx.doi. org/10.1016/j.jtbi.2013.12.024 PMID:24406808

17. Kucharski AJ, Althaus CL. The role of superspreading in Middle East respiratory syndrome coronavirus (MERS-CoV) transmission. Euro Surveill. 2015;20(25):21167. Available from: http://www.eurosurveillance.org/ViewArticle. aspx?Articleld=21167 PMID:26132768</jrn〉

18. 〈jrn〉18. Bauch CT, Oraby T. Assessing the pandemic potential of MERS-CoV. Lancet. 2013;382(9893):662-4. http://dx.doi. org/10.1016/S0140-6736(13)61504-4 PMID:23831143

19. Cowling BJ, Park M, Fang VJ, Wu P, Leung GM, Wu JT. Preliminary epidemiological assessment of MERSCoV outbreak in South Korea, May to June 2015. Euro Surveill. 2015;20(25):21163. Available from: http://www.
eurosurveillance.org/ViewArticle. aspx?Articleld $=21163$ PMID:26132767

20. Park HY, Lee EJ, Ryu YW, Kim Y, Kim H, Lee H, et al. Epidemiological investigation of MERS-CoV spread in a single hospital in South Korea, May to June 2015. Euro Surveill. 2015;20(25):21169. Available from: http://www. eurosurveillance.org/ViewArticle.aspx?Articleld=21169 PMID:26132766 